Supporting Information

\title{
Perovskite Ink with Ultra-Wide Processing Window for Efficient and Scalable Perovskite Solar Cells in Ambient Air
}

Jian Su, Hongkun Cai, * Jingtao Yang, Xiaofang Ye, Rui Han, Jian Ni, * Juan Li and Jianjun Zhang

Department of Electronic Science and Technology, College of Electronic Information and Optical Engineering, Nankai University, Tianjin, 300350, China

E-mail: caihongkun@,nankai.edu.cn, nijian_nankai@163.com 


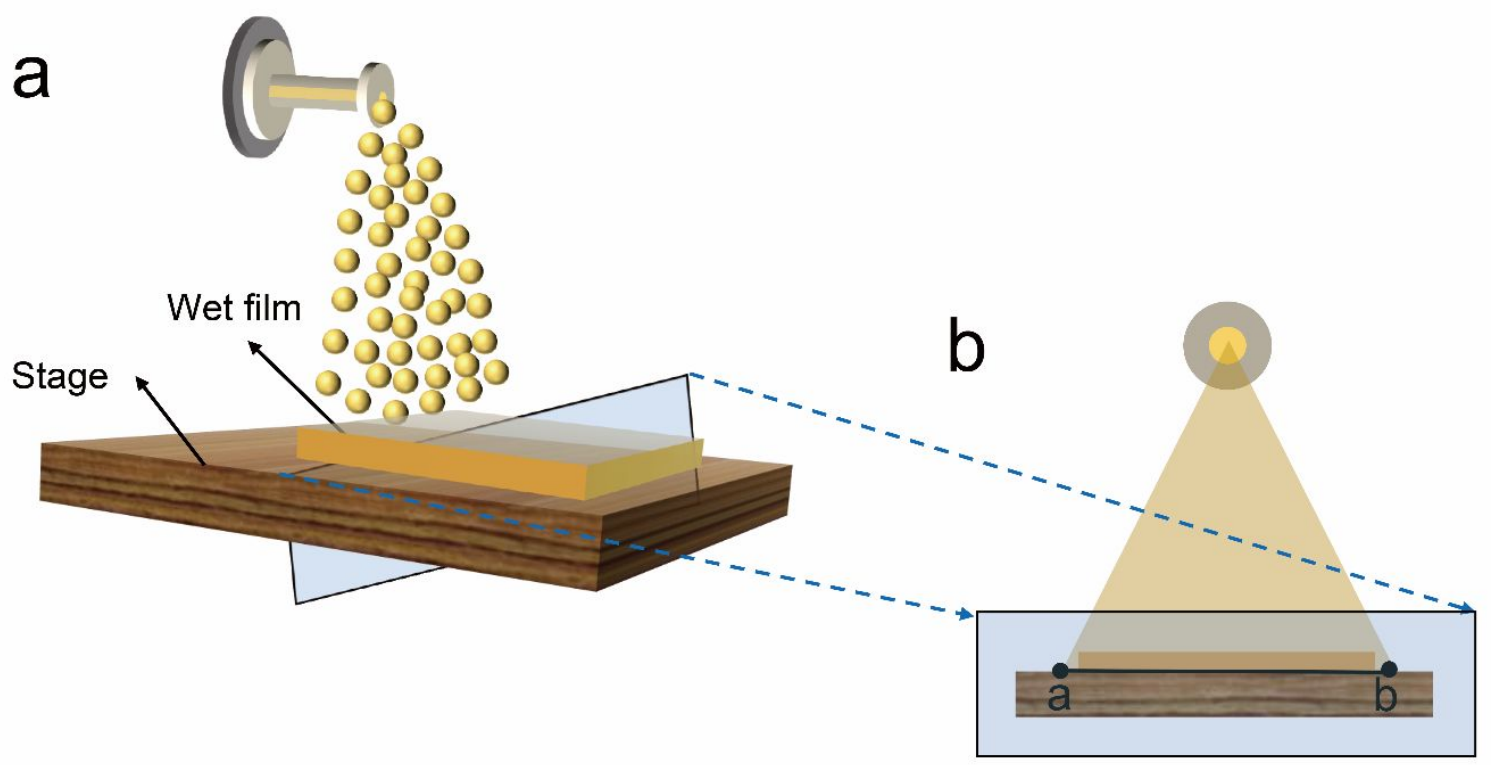

Figure S1. (a) Schematic illustration of spray coating. (b) Cross-section view of (a). $l_{a b}$ was the length of contact line between spray droplets and stage. 


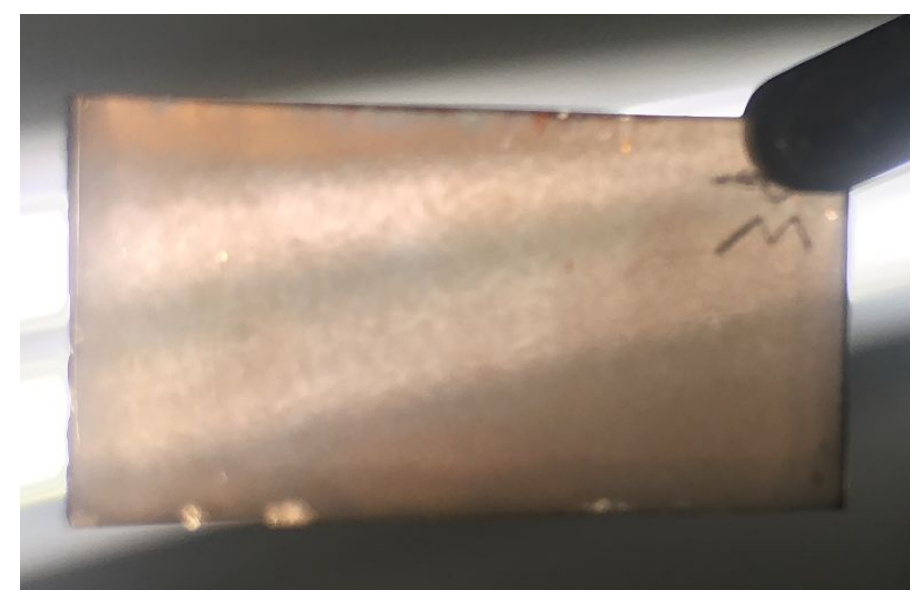

Figure S2. Optical photo of sprayed perovskite film without performing antisolvent. There is heterogeneous crysrallization occured in perovskite films with absence of antisolvent treatment, resulting in poor coverage of perovskite film. 


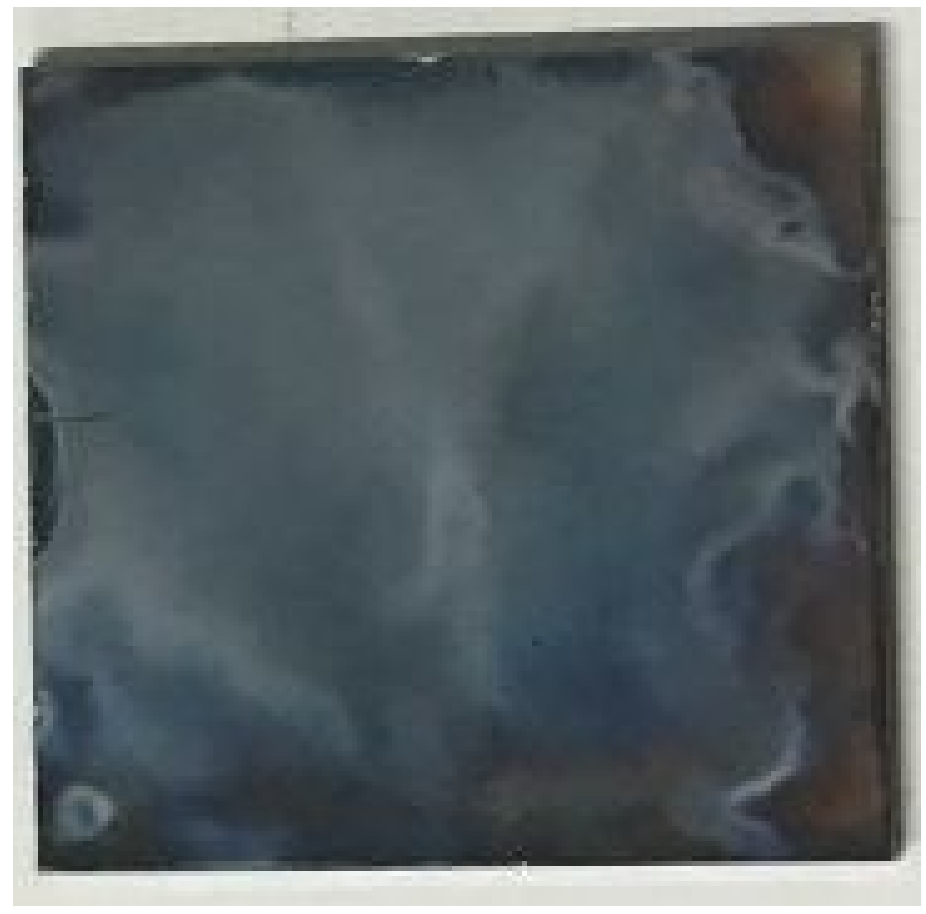

Figure S3. Optical photo of sprayed perovskite film prepared from a precursor ink with DMF \& DMSO. 

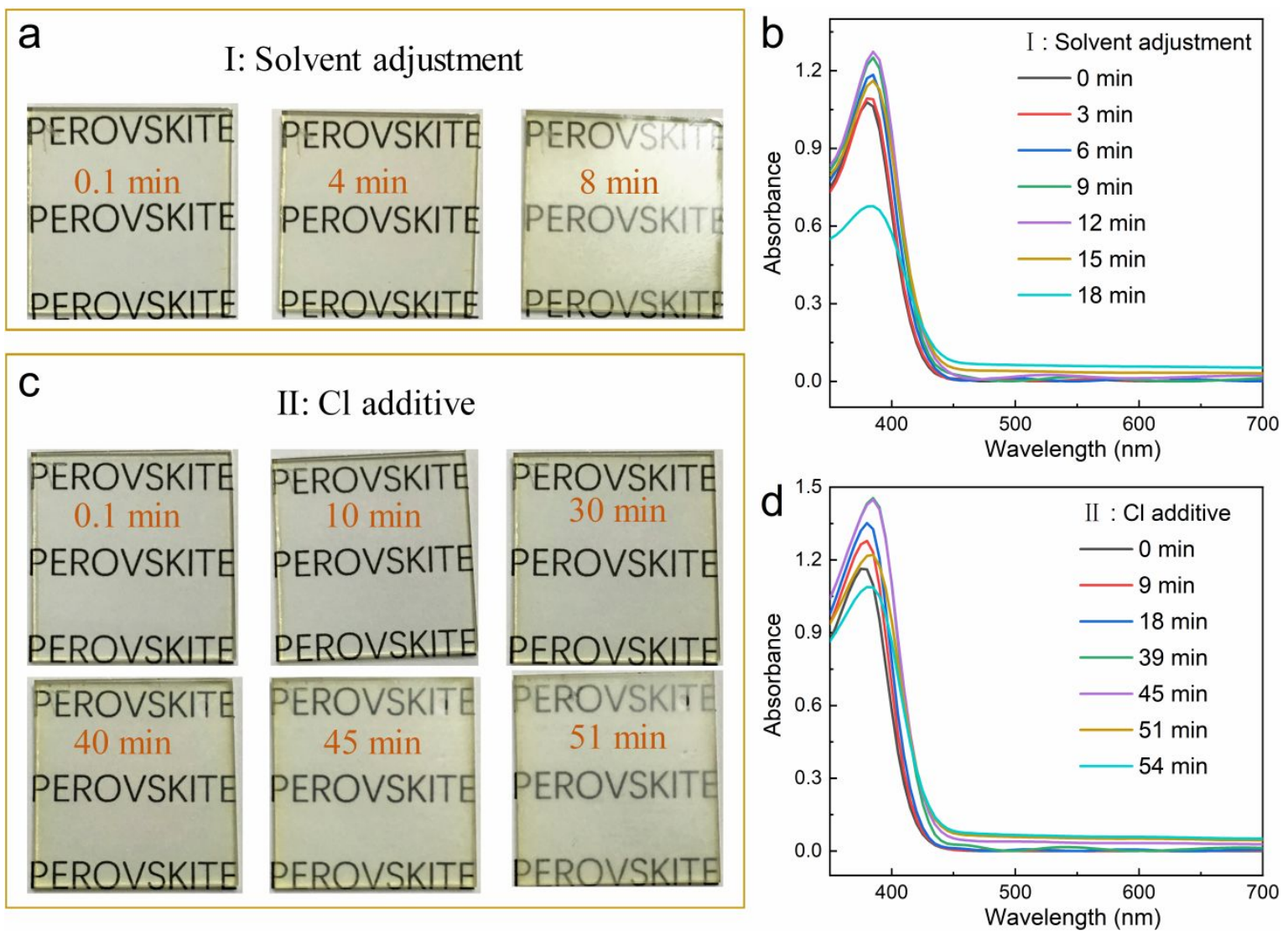

Figure S4. (a, c) Optical images from wet film (transparent film) to dry film (foggy film) and (b, d) in-suit absorption spectrum of sprayed perovskite wet films at various processing window extended by two different strategies. 

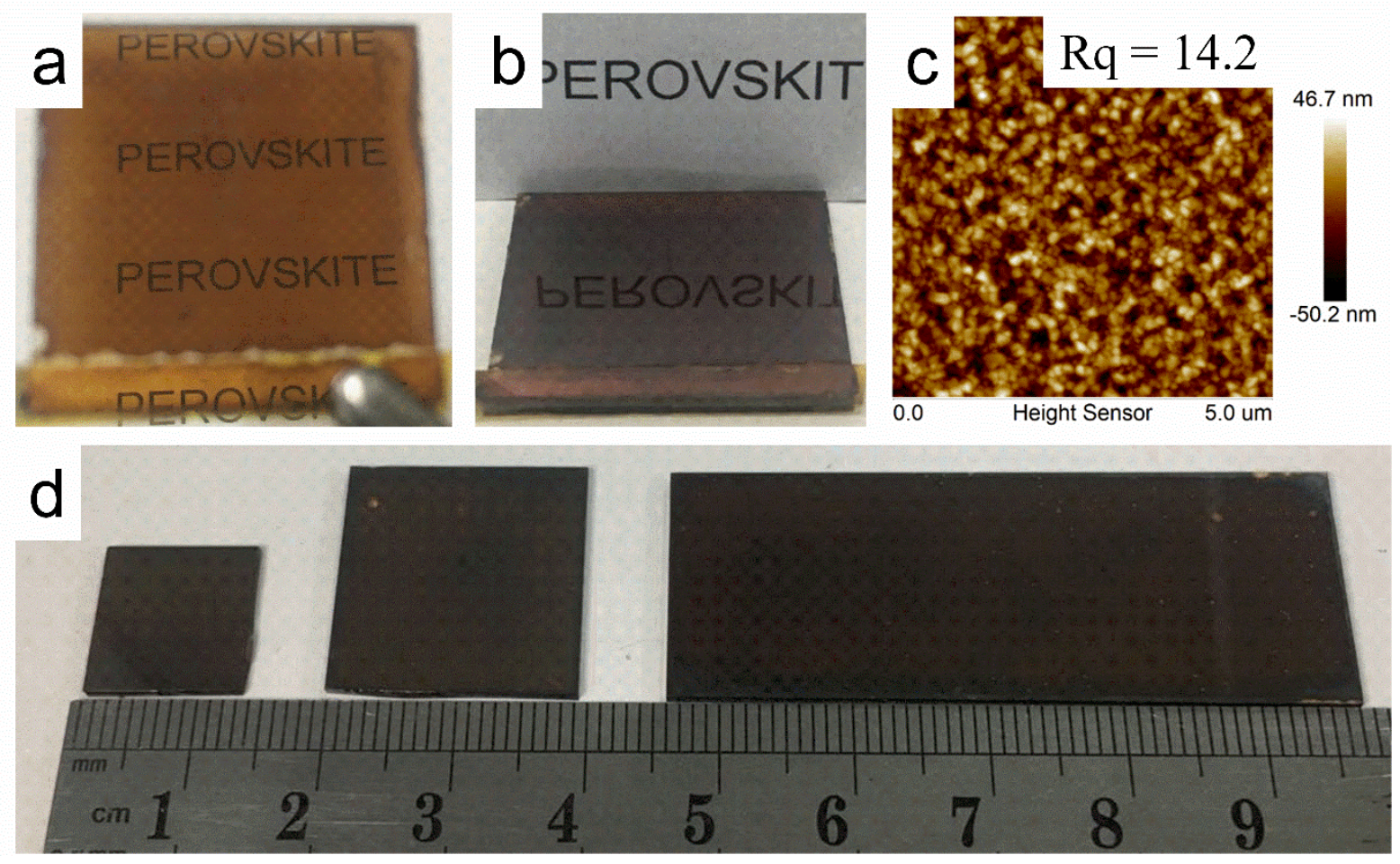

Figure S5. (a) Optical photo of semi-transparent sprayed perovskite film. (b) Optical photo of a mirror-like sprayed perovskite film. (c) AFM image of the sprayed perovskite film with a Rq of $14.2 \mathrm{~nm}$. (d) Optical photos of sprayed perovskite films on different size substrate. 

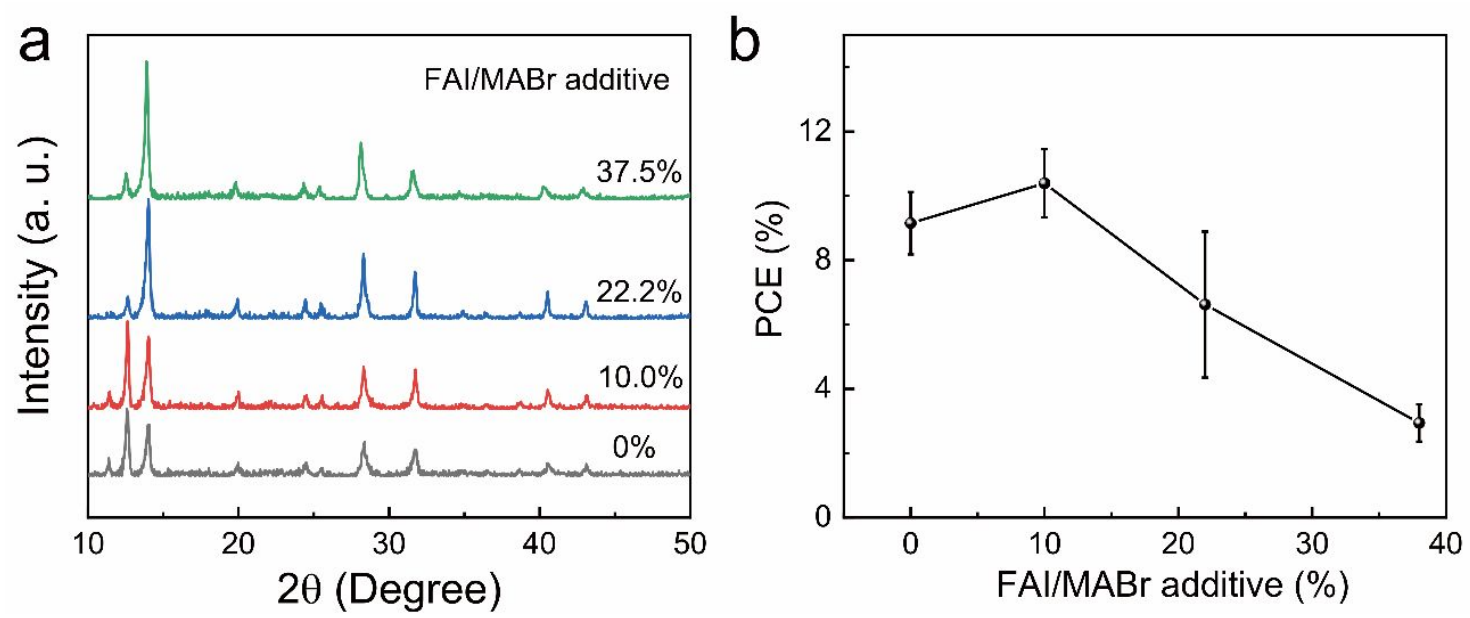

Figure S6. FAI/MABr additive. (a) XRD diffraction pattern of perovskite films with different amounts of FAI/MABr additive. (b) Corresponding PCE of the PSCs with different amounts of FAI/MABr additive. 


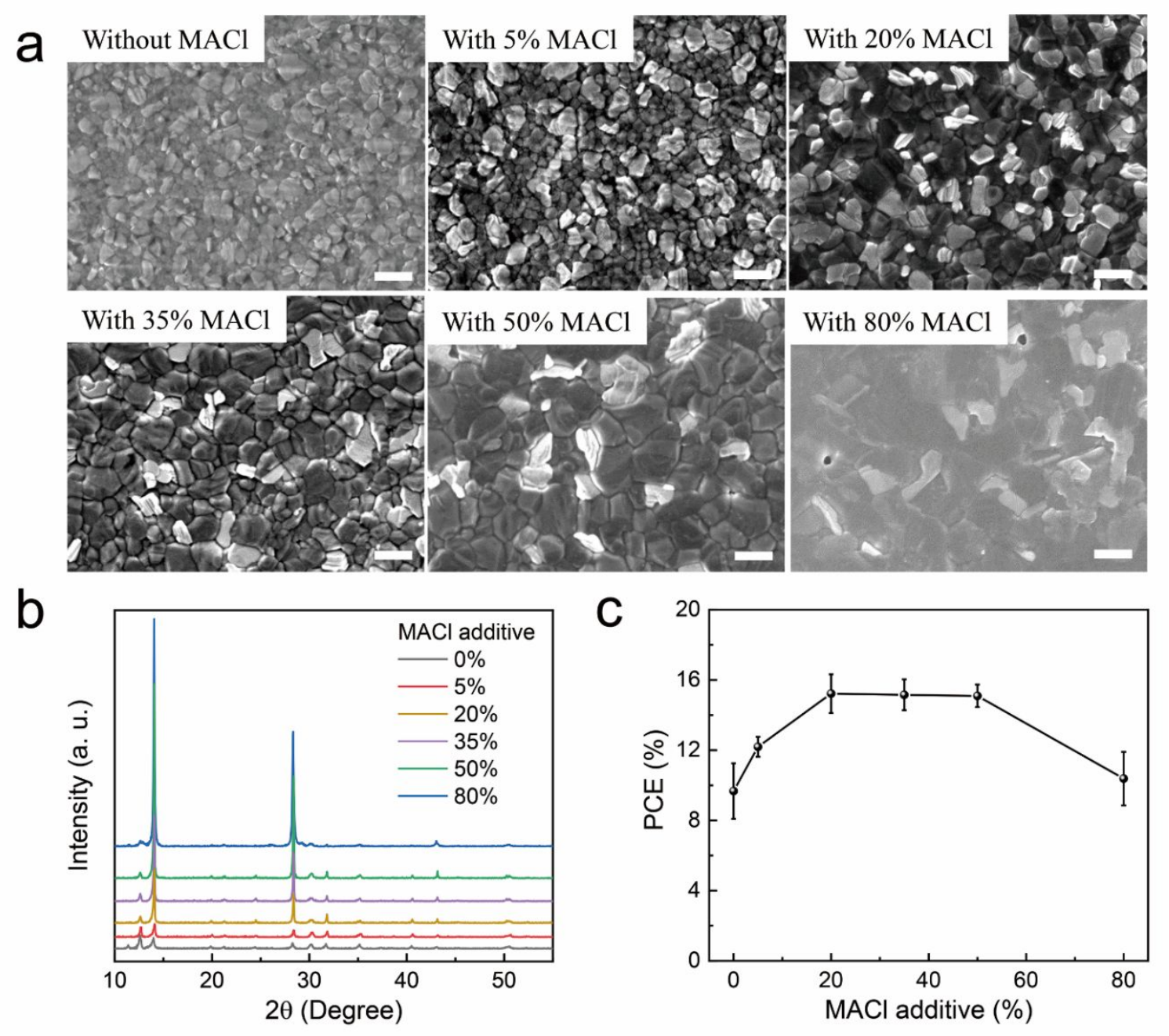

Figure S7. MACI additive. (a) SEM images, (b) XRD diffraction pattern of perovskite films with different amount of $\mathrm{MACl}$ additive. (c) Corresponding PCE of the PSCs with different amount of MACl additive. 


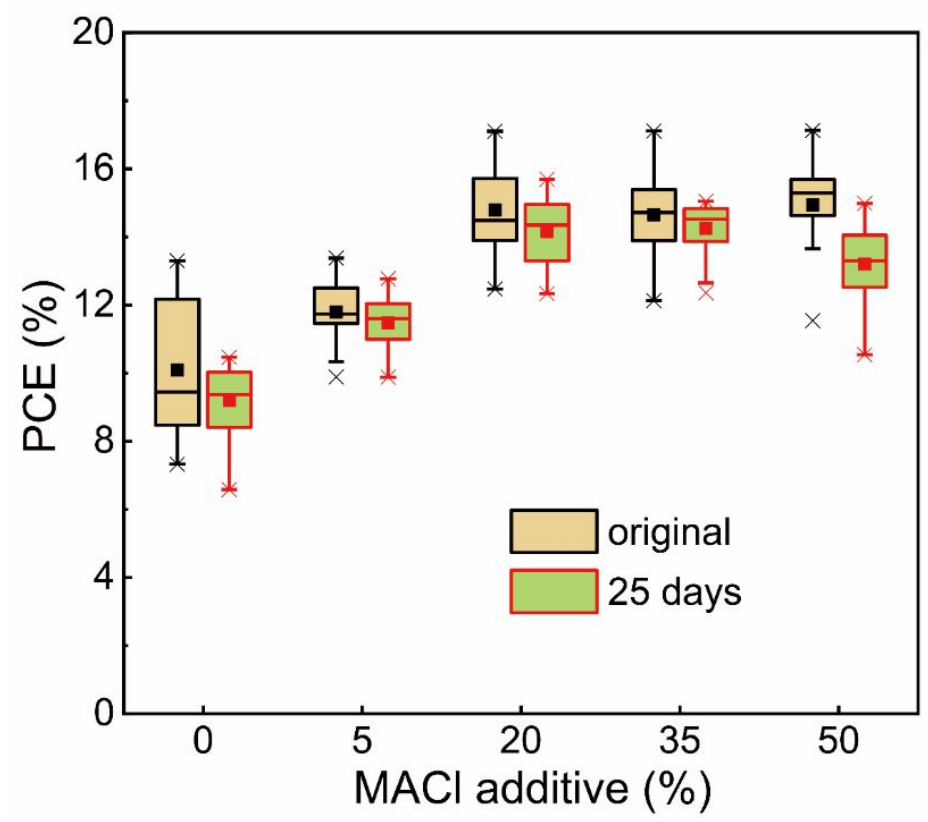

Figure S8. Device stability. The comparison of efficiency between the original devices and the devices after 25 days with different amount of $\mathrm{MACl}$ additive (shelf storage condition: dark and dry air, RH $10 \sim 20 \%$ ). 


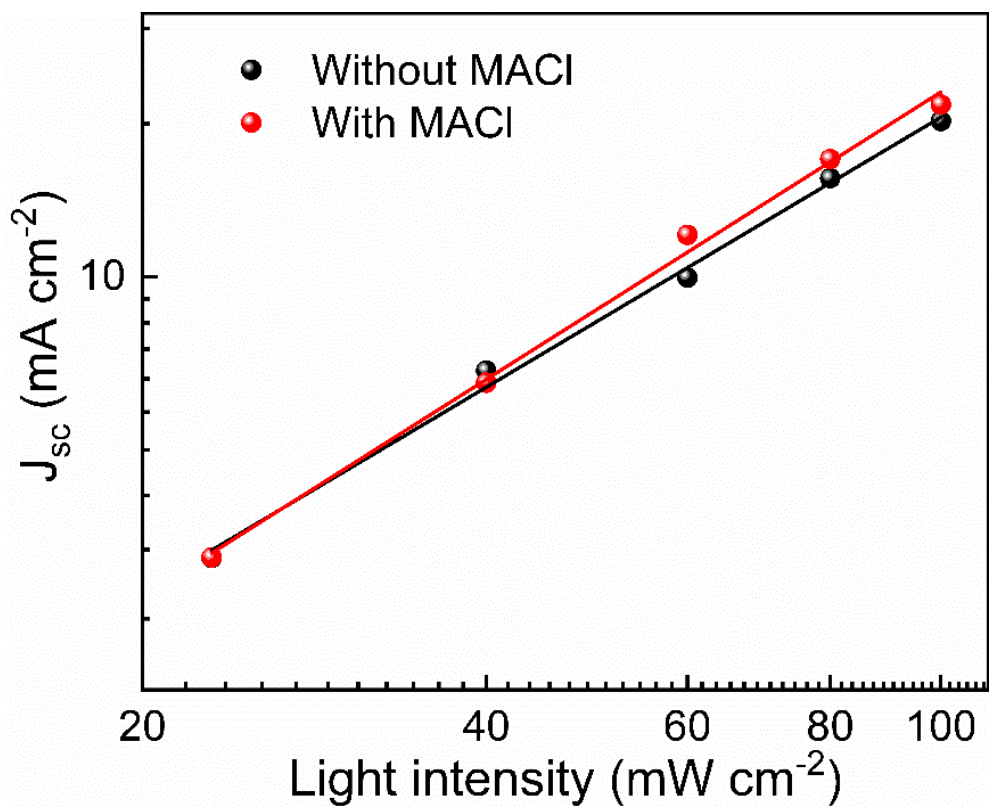

Figure S9. Current density $\left(J_{s c}\right)$ vs. light intensity for the PSCs without and with $\mathrm{MACl}$. 

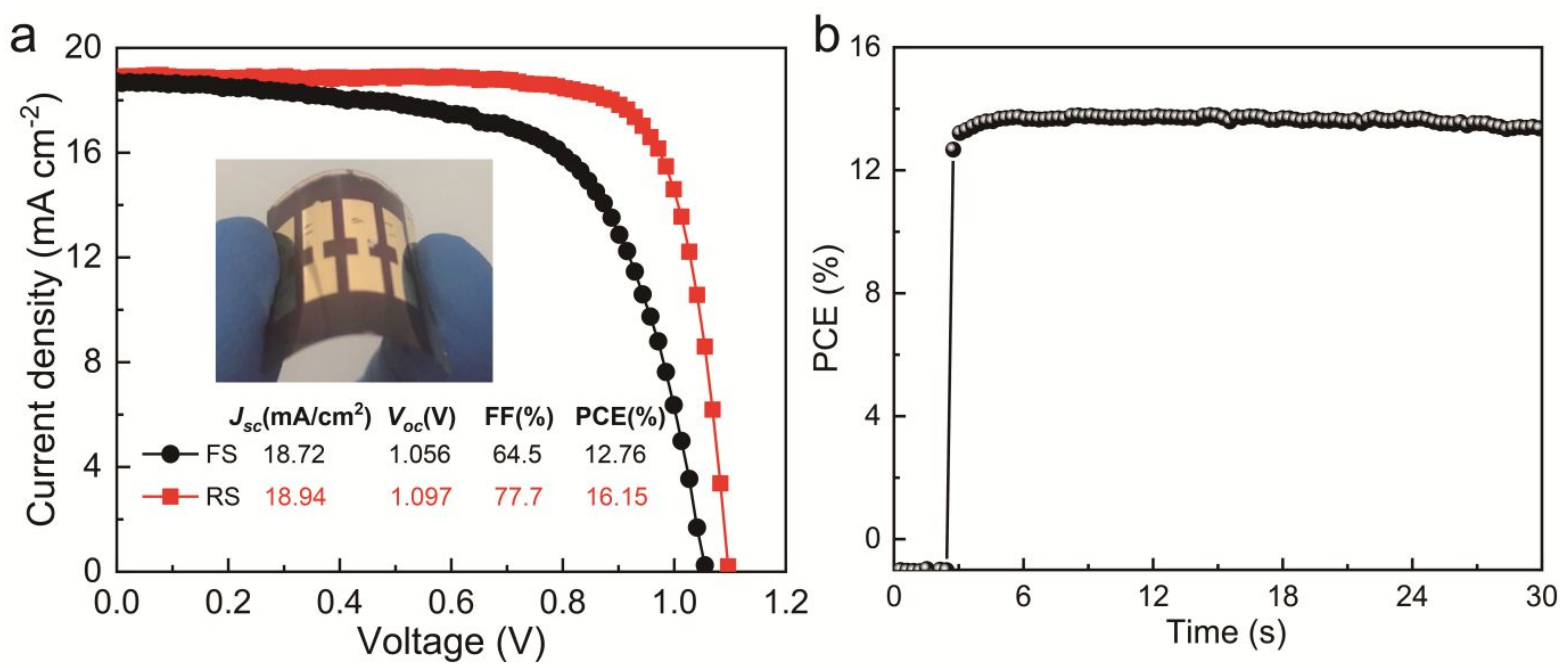

Figure S10 (a) $J-V$ curves of champion flexible device based on reverse scan and forward scan. The inset photograph was the flexible devices with an area of $0.29 \mathrm{~cm}^{2}$ (without etched ITO/PET). (b) Stabilized power output of the flexible device of (a). 


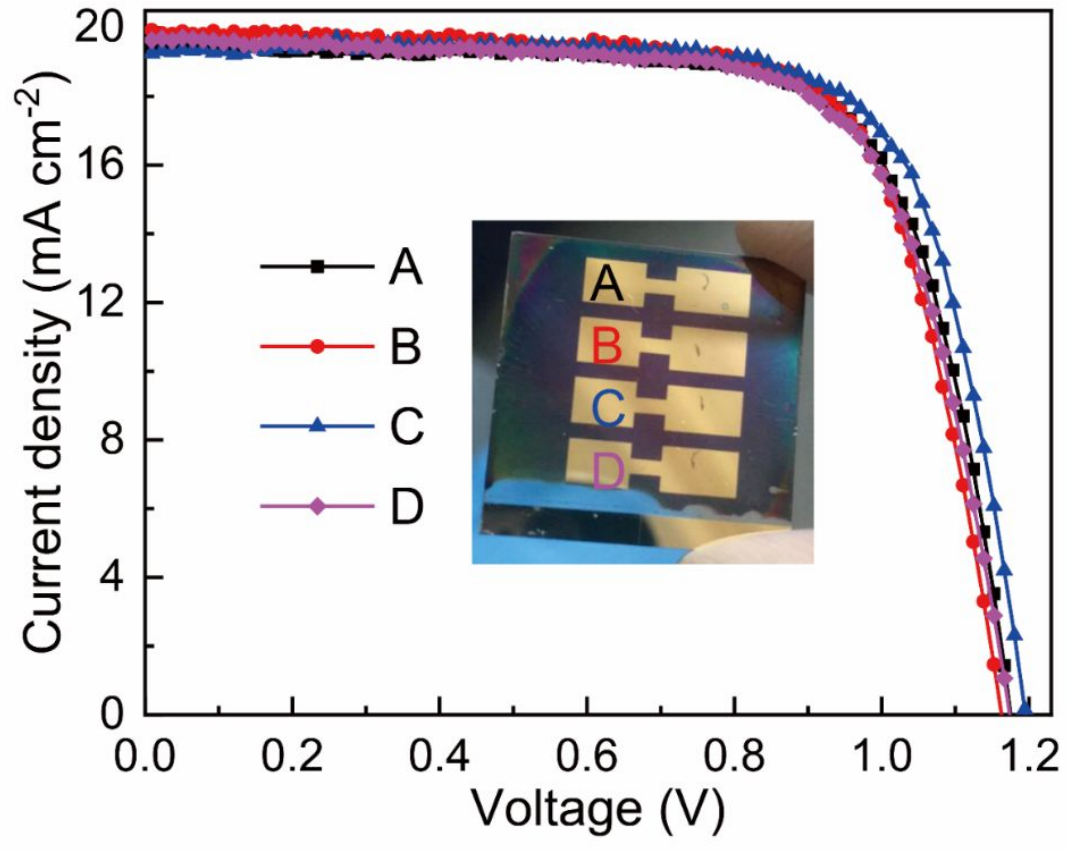

Figure S11. Photograph and $J-V$ curves of four individual cells on the $2 \times 2 \mathrm{~cm}$ substrate. 
Table S1. Typical physical parameters of common solvents used for perovskite inks.

\begin{tabular}{cccccc}
\hline Solvent & $\begin{array}{c}\text { Density } \\
(\mathbf{g} / \mathbf{m l})\end{array}$ & $\begin{array}{c}\text { Boiling point } \\
\left({ }^{\circ} \mathbf{C}\right)\end{array}$ & $\begin{array}{c}\text { Vapor Pressure } \\
\left(\mathbf{m m H g}, \mathbf{2 0}{ }^{\circ} \mathrm{C}\right)\end{array}$ & $\begin{array}{c}\text { Surface tension } \\
(\mathbf{d y n e} / \mathbf{c m})\end{array}$ & $\begin{array}{c}\text { Viscosity } \\
(\mathbf{m P a})\end{array}$ \\
\hline DMF & 0.948 & 153 & 2.7 & 25.75 & 0.92 \\
DMSO & 1.100 & 189 & 0.42 & 43.6 & 2 \\
NMP & 1.028 & 203 & 0.29 & 33.75 & 1.67 \\
GBL & 1.129 & 204 & 1.5 & 35.45 & 1.7 \\
\hline
\end{tabular}


Table S2. The power conversion efficiency (PCE) of sprayed perovskite solar cells (PSCs) by using the precursor ink with different ratios of binary solvent (DMF: NMP).

\begin{tabular}{ccccc}
\hline DMF:NMP (v:v) & $\boldsymbol{J}_{\boldsymbol{s c}}\left(\mathbf{m A} / \mathbf{c m}^{2}\right)$ & $\boldsymbol{V}_{\boldsymbol{o c}}(\mathbf{V})$ & FF (\%) & PCE (\%) \\
\hline $1: 1$ & $12.84 \pm 0.64$ & $0.92 \pm 0.07$ & $34.29 \pm 4.82$ & $4.08 \pm 0.94$ \\
$2: 1$ & $18.27 \pm 0.48$ & $1.15 \pm 0.03$ & $54.77 \pm 1.14$ & $11.51 \pm 0.32$ \\
$4: 1$ & $18.20 \pm 0.48$ & $1.09 \pm 0.01$ & $56.52 \pm 3.16$ & $11.20 \pm 0.71$ \\
$8: 1$ & $16.52 \pm 0.40$ & $1.06 \pm 0.03$ & $52.74 \pm 2.03$ & $9.25 \pm 0.59$ \\
\hline
\end{tabular}


Table S3. $J-V$ parameters of champion devices without and with MACl additive.

\begin{tabular}{ccccccc}
\hline Champion cell & & $\boldsymbol{J}_{s c}\left(\mathbf{m A} / \mathbf{c m}^{2}\right)$ & $\boldsymbol{V}_{\boldsymbol{c}}(\mathbf{V})$ & FF (\%) & PCE (\%) $\begin{array}{l}\text { Hysteresis } \\
\text { index (\%) }\end{array}$ \\
\hline Without MACl & RS & 18.68 & 1.154 & 63.5 & 13.69 \\
& FS & 18.17 & 1.124 & 55.2 & 11.26 \\
With MACl & RS & 20.54 & 1.195 & 75.5 & 18.53 \\
& FS & 20.53 & 1.172 & 67.9 & 16.34 \\
\hline
\end{tabular}


Table S4. The comparison between our work and previous work of sprayed perovskite solar cells.

\begin{tabular}{|c|c|c|c|c|c|c|c|}
\hline \multirow{3}{*}{$\begin{array}{l}\text { Perovskite } \\
\text { composition }\end{array}$} & \multirow{3}{*}{$\begin{array}{c}\text { Spray } \\
\text { condition }\end{array}$} & \multirow{3}{*}{$\begin{array}{c}\text { Treatment of } \\
\text { Wet film }\end{array}$} & \multicolumn{4}{|c|}{ PCE $(\%)$} & \multirow{3}{*}{$\begin{array}{c}\text { Published } \\
\text { work }\end{array}$} \\
\hline & & & \multicolumn{2}{|c|}{ Rigid PSCs } & \multicolumn{2}{|c|}{ Flexible PSCs } & \\
\hline & & & $<1 \mathrm{~cm}^{2}$ & $>1 \mathrm{~cm}^{2}$ & $<1 \mathrm{~cm}^{2}$ & $>1 \mathrm{~cm}^{2}$ & \\
\hline $\mathrm{MAPbI}_{3-\mathrm{x}} \mathrm{Cl}_{\mathrm{x}}$ & Air & $\begin{array}{l}\text { substrate } \\
\text { heating }^{\mathrm{a}}\end{array}$ & 11.10 & - & - & - & 1 \\
\hline $\mathrm{MAPbI}_{3-\mathrm{x}} \mathrm{Cl}_{\mathrm{x}}$ & Air & $\begin{array}{l}\text { substrate } \\
\text { heating }\end{array}$ & 13.00 & - & 8.10 & - & 2 \\
\hline $\mathrm{MAPbI}_{3}$ & Air & $\begin{array}{l}\text { substrate } \\
\text { heating }\end{array}$ & 16.03 & 13.09 & - & - & 3 \\
\hline $\mathrm{MAPbI}_{3-\mathrm{x}} \mathrm{Cl}_{\mathrm{x}}$ & Air & $\begin{array}{l}\text { substrate } \\
\text { heating }\end{array}$ & 18.30 & 15.50 & - & - & 4 \\
\hline $\mathrm{MAPbI}_{3}$ & Air & antisolvent $^{\mathrm{a}}$ & 17.29 & - & - & - & 5 \\
\hline $\mathrm{MAPbI}_{3}$ & Air & $\begin{array}{c}\text { Plasma } \\
\text { Processing }\end{array}$ & 15.70 & - & - & - & 6 \\
\hline CsFAMA $^{c}$ & $\mathrm{~N}_{2}$ & vacuum flash ${ }^{\mathrm{a}}$ & 17.80 & - & - & - & 7 \\
\hline $\mathrm{MAPbI}_{3-\mathrm{x}} \mathrm{Cl}_{\mathrm{x}}$ & Air & hot air blowing ${ }^{a}$ & 13.50 & 9.80 & - & 6.71 & 8 \\
\hline $\operatorname{CsFAMA}^{c}$ & Air & antisolvent $^{\mathbf{a}}$ & 18.53 & 15.07 & 16.15 & 13.21 & This work \\
\hline
\end{tabular}

${ }^{\text {a) }}$ one-step method; b)two-step method; ${ }^{\mathrm{c})} \mathrm{CsMAFA}$ is $\mathrm{CsI}_{0.05}\left(\left(\mathrm{FAPbI}_{3}\right)_{0.85}\left(\mathrm{MAPbBr}_{3}\right)_{0.15}\right)_{0.95}$. 


\section{References}

(1) Barrows, A. T.; Pearson, A. J.; Kwak, C. K.; Dunbar, A. D. F.; Buckley, A. R.; Lidzey, D. G., Efficient Planar Heterojunction Mixed-Halide Perovskite Solar Cells Deposited via Spray-Deposition. Energy \& Environ. Sci. 2014, 7 (9), 2944-2950.

(2) Das, S.; Yang, B.; Gu, G.; Joshi, P. C.; Ivanov, I. N.; Rouleau, C. M.; Aytug, T.; Geohegan, D. B.; Xiao, K., High-Performance Flexible Perovskite Solar Cells by Using a Combination of Ultrasonic Spray-Coating and Low Thermal Budget Photonic Curing. ACS Photonics 2015, 2 (6), 680-686.

(3) Huang, H.; Shi, J.; Zhu, L.; Li, D.; Luo, Y.; Meng, Q., Two-Step Ultrasonic Spray Deposition of CH3NH3PbI3 For Efficient and Large-Area Perovskite Solar Cell. Nano Energy 2016, 27, 352-358.

(4) Heo, J. H.; Lee, M. H.; Jang, M. H.; Im, S. H., Highly Efficient CH3NH3PbI3-xClx Mixed Halide Perovskite Solar Cells Prepared by Re-Dissolution and Crystal Grain Growth via Spray Coating. J. Mater. Chem. A 2016, 4 (45), 17636-17642.

(5) Bishop, J. E.; Smith, J. A.; Greenland, C.; Kumar, V.; Vaenas, N.; Game, O. S.; Routledge, T. J.; WongStringer, M.; Rodenburg, C.; Lidzey, D. G., High-Efficiency Spray-Coated Perovskite Solar Cells Utilizing Vacuum-Assisted Solution Processing. ACS Appl. Mater. \& Interfaces 2018, 10 (46), 3942839434.

(6) Hilt, F.; Hovish, M. Q.; Rolston, N.; Brüning, K.; Tassone, C. J.; Dauskardt, R. H., Rapid Route to Efficient, Scalable, and Robust Perovskite Photovoltaics in Air. Energy \& Environ. Sci. 2018, 11 (8), 2102-2113.

(7) Uličná, S.; Dou, B.; Kim, D. H.; Zhu, K.; Walls, J. M.; Bowers, J. W.; van Hest, M. F. A. M., Scalable Deposition of High-Efficiency Perovskite Solar Cells by Spray-Coating. ACS Appl. Energy Mater. 2018, 1 (5), 1853-1857.

(8) Su, J.; Cai, H.; Ye, X.; Zhou, X.; Yang, J.; Wang, D.; Ni, J.; Li, J.; Zhang, J., Efficient Perovskite Solar Cells Prepared by Hot Air Blowing to Ultrasonic Spraying in Ambient Air. ACS Appl. Mater. \& Interfaces 2019, 11 (11), 10689-10696. 